\title{
Gerechte Apfelteilung - keine leichte Aufgabe!
}

\author{
Hans Humenberger
}

Eingegangen: 5. September 2017 / Angenommen: 2. Oktober 2017 / Online publiziert: 3. November 2017 (C) The Author(s) 2017. Dieser Artikel ist eine Open-Access-Publikation.

Zusammenfassung Dieser Aufsatz bezieht sich stark auf einen 2015 in den Semesterberichten publizierten Aufsatz („Pizza-Theorem“). Ohne weitere formale Betrachtungen und langwierige Berechnungen gelingt eine Verallgemeinerung auf eine räumliche Situation. Ein wesentliches Mittel dabei ist das berühmte Cavalieri-Prinzip.

Das wirklich gerechte Teilen eines Apfels (bzw. einer Melone, Kartoffel, etc.) ist - genau genommen - gar nicht so einfach, und zwar schon dann, wenn nur zwei Personen beteiligt sind (und man den Apfel - bzw. die Melone oder Kartoffel klassischerweise als kugelförmig annimmt). Mit einem geraden Messer muss man dabei immerhin den Mittelpunkt treffen, so dass der Schnitt ein „Großkreis“ ist. Näherungsweise klappt das sicher gut, i. A. ist wohl kein Streit nach der Teilung zu erwarten. Aber was ist, wenn man es wirklich genau machen will? Solche Überlegungen haben naturgemäß eher theoretischen als praktischen Charakter, können aber dennoch mathematisch und fachdidaktisch sehr wertvoll sein. In der Mathematik geht es eben nicht nur um Praxis, sondern auch um Theorie.

Mit dazu genau analogen Worten beginnt ein Aufsatz über die gerechte Pizzateilung („Pizza-Theorem“) - Humenberger 2015. Nun sind wir eine Dimension höher, also im Raum, eine Kugel statt eines Kreises. In dieser kurzen Note wird es darum gehen, aus dem ,ebenen“ Pizza-Theorem zu ernten und daraus unmittelbar eine interessante Verallgemeinerung auf den Raum abzulesen. Wir brauchen dafür keine abstrakt-formalen Betrachtungen oder langwierige Berechnungen durchzuführen, sondern müssen im Wesentlichen nur das berühmte Cavalieri-Prinzip anwenden.

H. Humenberger $(\bowtie)$

Wien, Österreich

E-Mail: hans.humenberger@univie.ac.at 

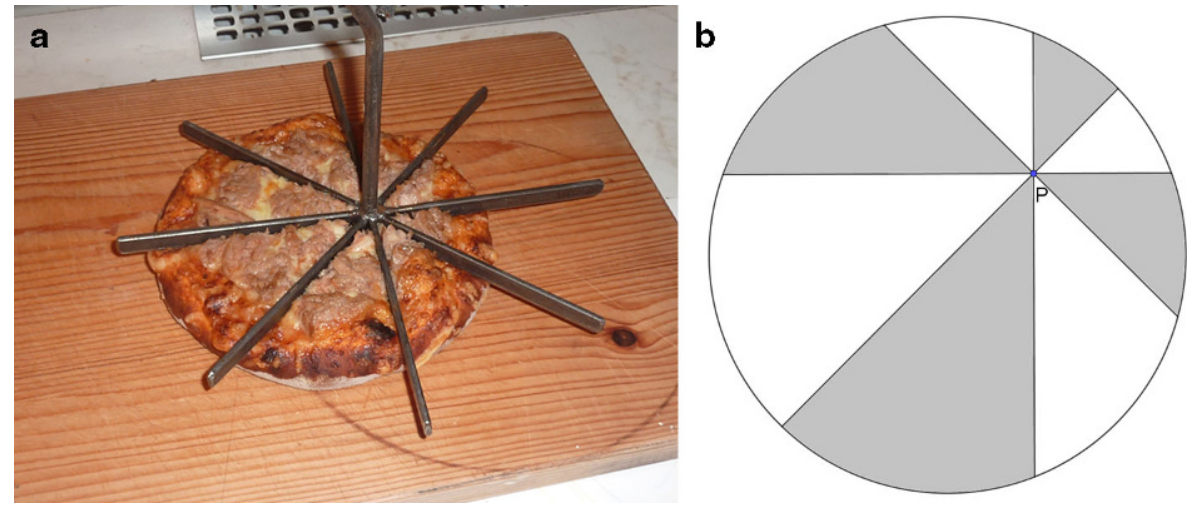

Abb. 1 a Pizzateilung, b Stanze schematisch

Ich bin auf diese Verallgemeinerung gekommen, weil mich B. Schuppar (Dortmund) auf eine Aufgabe aus dem Bundeswettbewerb Mathematik aufmerksam gemacht hat (2008, 2. Runde):

Aufgabe 3 Durch einen inneren Punkt einer Kugel werden drei paarweise aufeinander senkrecht stehende Ebenen gelegt. Diese zerlegen die Kugeloberfläche in acht krummlinige Dreiecke. Die Dreiecke werden abwechselnd schwarz und weiß so gefärbt, dass die Oberfläche der Kugel schachbrettartig aussieht. ist.

Man beweise, dass dann genau die Hälfte der Kugeloberfläche schwarz gefärbt

Das hat mich irgendwie sofort an das Pizza-Theorem erinnert. Könnte man mit der in Humenberger 2015 beschriebenen „Pizzastanze“ - mit $45^{\circ}$ zwischen benachbarten Stanzmessern - vielleicht auch eine Kugel (Apfel, Melone, Kartoffel, etc.) gerecht teilen?

Das überraschende Pizza-Theorem besagt, dass unabhängig von der Lage von $P$ (Abb. 1b) die grauen Flächen zusammen immer genau so groß sind wie die weißen zusammen, nämlich die halbe Kreisfläche (und auch die Summen der Pizzaränder sind bei Grau und Weiß gleich, nämlich der halbe Kreisumfang).

Wenn man sich nun vorstellt, dass man einen kugelförmigen Apfel mit einer solchen Stanze zerteilt (die Stanzachse muss dabei nicht notwendig durch den $\mathrm{Ku}$ gelmittelpunkt gehen, sondern kann den Apfel auch „,dezentral“ treffen), erhält man 8 keilförmige „Apfelspalten“ (bei Kartoffeln spricht man dabei oft von „wedges“). Man kann sich nun wieder vorstellen, jede zweite „Spalte“ grau bzw. weiß zu färben und erhält dann - auf die Ebene projiziert - das analoge Bild wie bei der Pizzateilung.

Als räumliches Bild ergibt sich dabei so etwas wie in Abb. 2.

$A$ und $B$ markieren dabei jene Punkte, in denen das Zentrum der Stanze, also die die Stanzachse, die Kugel „,betritt“ bzw. diese ,,verlässt“. Von oben betrachtet läge in der ebenen Projektion von oben dort genau der Punkt $P$. Nun kann man in nahe liegender Weise nach dem dreidimensionalen Analogon des Pizza-Theorems fragen: Sind die Summen der Volumina (bzw. Oberflächeninhalte) von Grau und Weiß wie- 
Abb. 2 Teilung eines Apfels in keilförmige Spalten (,wedges"), jeweils $45^{\circ}$

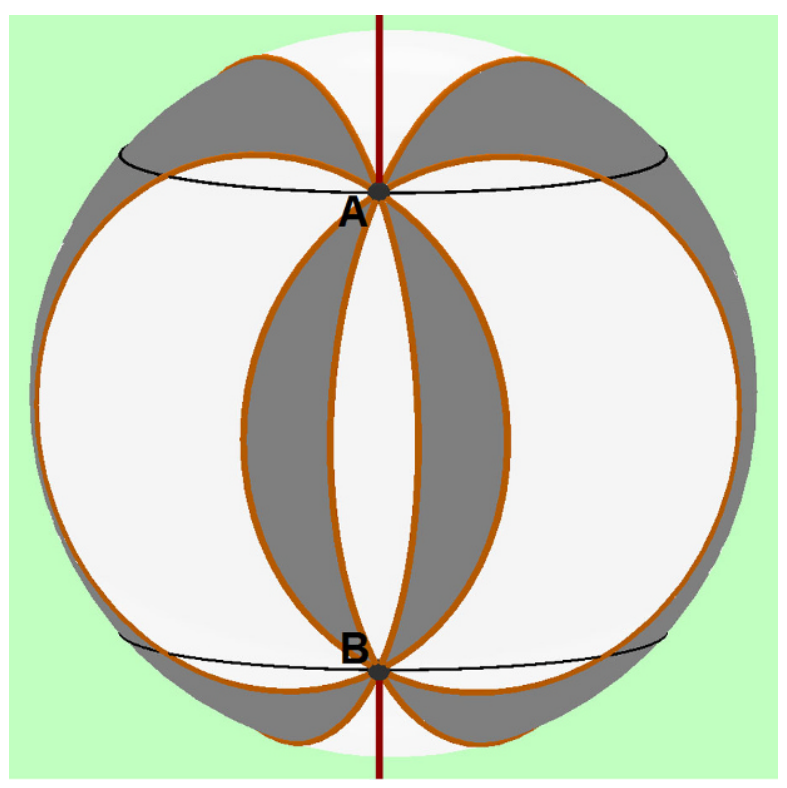

der gleich? Manchmal? Immer? Nie? A priori ist klar: Wenn ein Stanzmesser durch den Kugelmittelpunkt schneidet, dann ist die Gleichheit von Grau und Weiß aus Symmetriegründen gegeben. Aber wenn man schon sehr weit an die Peripherie geht mit dem Stanzzentrum (d.h. wenn $A$ und $B$ in Abb. 2 nur wenig übereinander liegen), dann ist es schon ziemlich klar, dass die in Abb. 2 großteils gar nicht sichtbare weiße Fläche „hinten“ schon ziemlich groß, also mehr als die halbe Kugeloberfläche weiß sein wird.

Das ist zunächst vielleicht einmal eine Enttäuschung. Denn immerhin kann man ja bei allen waagrechten ${ }^{1}$ Schnitten zwischen den beiden "Breitenkreisen“" von $A$ und $B$ eine Konstellation wie beim ebenen Pizza-Theorem feststellen, und dort herrscht ja bekanntlich immer Flächengleichheit zwischen Grau und Weiß. Nach dem Prinzip von Cavalieri ${ }^{2}$ folgt daraus unmittelbar die Volumengleichheit zwischen Grau und Weiß für die „Kugelschicht“ zwischen diesen beiden Breitenkreisen. Mit einem zum Pizza-Theorem analogen Argument (siehe Anhang) folgt wieder: In der Kugelschicht zwischen den beiden angesprochenen Breitenkreisen herrscht auch Flächengleichheit zwischen Grau und Weiß. Das Scheitern liegt also irgendwie an den beiden „Polen 3 “, also „,nördlich“ des Breitenkreises von $A$ und „,südlich“ des Breitenkreises von $B$. Diese beiden „Polregionen“ sind genau symmetrisch zur „Äquatorebene“, d. h. das, was nördlich von $A$ grau ist, ist auch südlich von $B$ grau

\footnotetext{
${ }^{1}$ Hiermit ist parallel zur Auflageebene des Apfels gemeint.

2 Dieses besagt bekanntlich: Zwei Körper besitzen dasselbe Volumen, wenn alle ihre Schnittflächen in Ebenen parallel zu einer Grundebene in entsprechenden Höhen den gleichen Flächeninhalt haben (vgl. [2, S. 468, 649]).

${ }^{3}$ Der Punkt des Apfels, mit dem er auf dem „Schneidbrett“ aufliegt, kann idealisiert als „Süidpol“ bezeichnet werden, der diametral gegenüberliegende Punkt als „Nordpol“.
} 

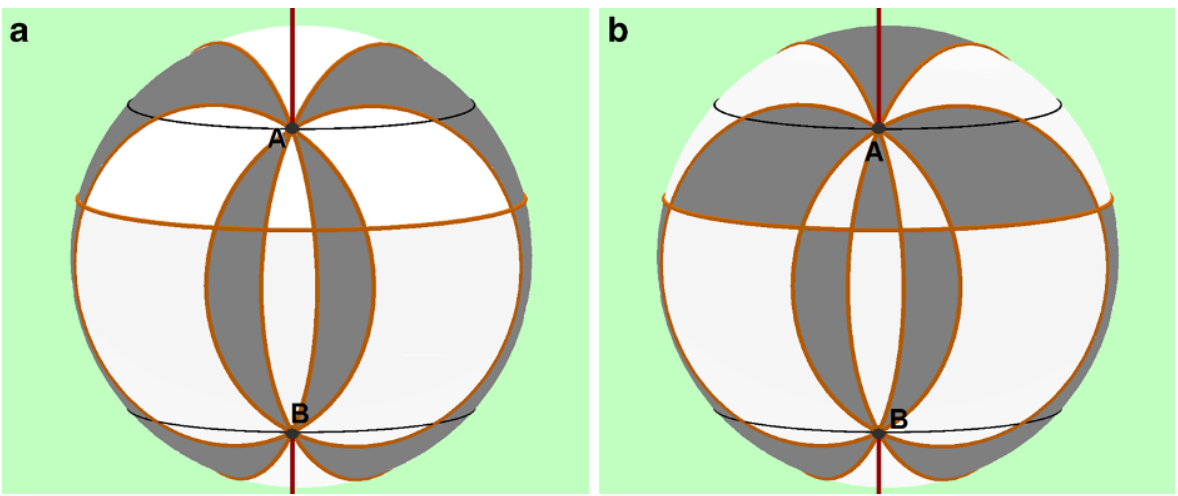

Abb. 3 a zusätzliche waagrechte Ebene. b Nach Umfärbung oben

und analog bei weiß. Für einen Ausgleich zwischen Grau und Weiß müsste also jede Polregion für sich schon ausgeglichen sein, aber ist das so?

Man braucht sich jedoch gar nicht lang den Kopf zu zerbrechen, ob es schon innerhalb der einzelnen Polregionen einen Ausgleich gibt (außer in den trivialen Fällen, in denen jede Polregion in sich symmetrisch aufgebaut ist in grau und weiß - wenn ein Stanzmesser durch den Kugelmittelpunkt geht), denn mit einer weiteren waagrechten Schnittebene irgendwo zwischen den beiden Breitenkreisen von $A$ und $B$ - dabei wird also jede „Apfelspalte“ einmal waagrecht geteilt, vgl. Abb. 3a kann man diesen Ausgleich erzwingen: Durch Umfärben aller Flächen auf dem oberen (oder auch unteren) Teil hat man klarerweise eine Polregion farblich genau „umgepolt“, so dass perfekter Ausgleich zwischen den beiden Polregionen gegeben ist (und zwischen den beiden Breitenkreisen herrscht wegen des Cavalieri-Prinzips natürlich immer noch Ausgleich zwischen Grau und Weiß), vgl. Abb. 3b.

Eine mögliche gerechte Teilung eines Apfels (Melone, Kartoffel) wäre also durch folgende Technik möglich (ohne den Mittelpunkt genau treffen zu müssen): Teile den Apfel zunächst mittels eines (fast beliebigen) waagrechten Schnittes in zwei Teile, dieser kann, muss aber nicht durch den Mittelpunkt gehen. Wenn man nun die so geteilte Frucht mit einer $45^{\circ}$-Pizza-Stanze von oben so durchschneidet, dass das Stanzzentrum irgendwie den oberen Teil der Frucht trifft, so entstehen $2 \times 8=$ 16 Teile (geteilte „Spalten“ bzw. ,wedges“), die dann gerecht auf zwei Personen aufgeteilt werden können, wenn sich jede/r die richtigen Teile nimmt (grau, weiß). Jede/r hat dann auch gleich viel „Schale“ bzw. „Oberfläche“ (genauere Begründung im Anhang; das Kerngehäuse eines Apfels wird dabei i. A. nicht gerecht geteilt). Wichtig dabei ist, dass der erste Schnitt waagrecht erfolgt, und dass die Pizzastanze einerseits genau lotrecht ${ }^{4}$ auf den Apfelgedrückt wird und andererseits genaue $45^{\circ}$ Winkel hat. Das sind auch keine trivialen Voraussetzungen, aber man braucht eben keinen Mittelpunkt zu treffen. Insgesamt ist die Theorie hier aber ohnehin wichtiger als die „Praxis“.

\footnotetext{
4 Hiermit ist senkrecht zur Auflageebene des Apfels gemeint.
} 
Abb. 4 Gerechte Teilung einer Pizza mit 3 Teilen pro Quadrant

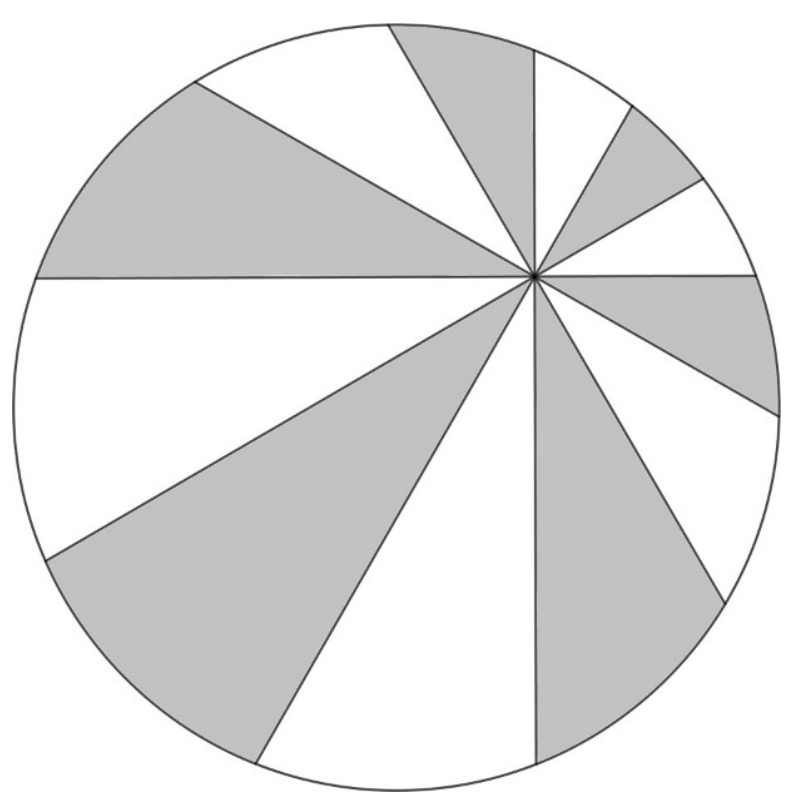

Wie beim ebenen Pizza-Theorem tritt auch hier - eine Dimension höher - ein interessantes Phänomen zu Tage: Ist man nur an einer gerechtenTeilung der Oberfläche interessiert, so reicht eine Stanze, die aus zwei zueinander orthogonalen Stanzmessern besteht (in der obigen Aufgabe vom Bundeswettbewerb Mathematik war die Rede von drei paarweise zueinander orthogonalen Ebenen, zwei davon repräsentieren im Stanzkontext die Stanzmesser, die dritte die oben erwähnte ,weitere waagrechte Schnittebene“). Will man auch eine gerechte Volumenteilung haben, so braucht man mindestens vier Stanzmesser (im $45^{\circ}$-Winkel), d.h. jeder Quadrant muss noch einmal halbiert werden. Dies ist deswegen so, weil man für das ebene Pizza-Theorem auch mindestens vier Stanzmesser (vgl. Abb. 1b) braucht, um die Konstanz der weißen bzw. bzw. grauen Flächensummen zu erreichen. Die Konstanz der Bogenlängensummen beim ebenen Pizza-Theorem ist schon mit zwei Stanzmessern zu erreichen (vgl. auch die Bemerkung in [3, S. 180]).

Das Pizza-Theorem gilt auch für andere Stanzmesser, bei denen jeder Quadrant in $3,4, \ldots$ gleichwinklige Teile geteilt wird (statt 8 also insgesamt 12, 16, $20 \ldots$ Pizzateile entstehen, siehe [3, S. $187 \mathrm{ff}$.]) - vgl. Abb. 4, dort mit drei Teilen pro Quadrant. Auch solche Stanzmesser kann man zur gerechten Teilung eines Apfels unter zwei Personen verwenden.

Dieser Aspekt lässt sich in natürlicher Weise auf die Apfelteilung übertragen. D. h. man kommt zu folgendem:

Apfel-Theorem Ein Apfel (bzw. eine Melone oder eine Kartoffel, mathematisch als Kugel modelliert) wird zunächst waagrecht einmal durchgeschnitten (das wird in Abb. 5 in Grund- und Aufriss dargestellt). Dann teilt man den Apfel noch mit einer „gleichwinkligen“ Stanze (jeder Quadrant ist dabei in gleich viele - mindestens 2 
Abb. 5 Apfel-Theorem

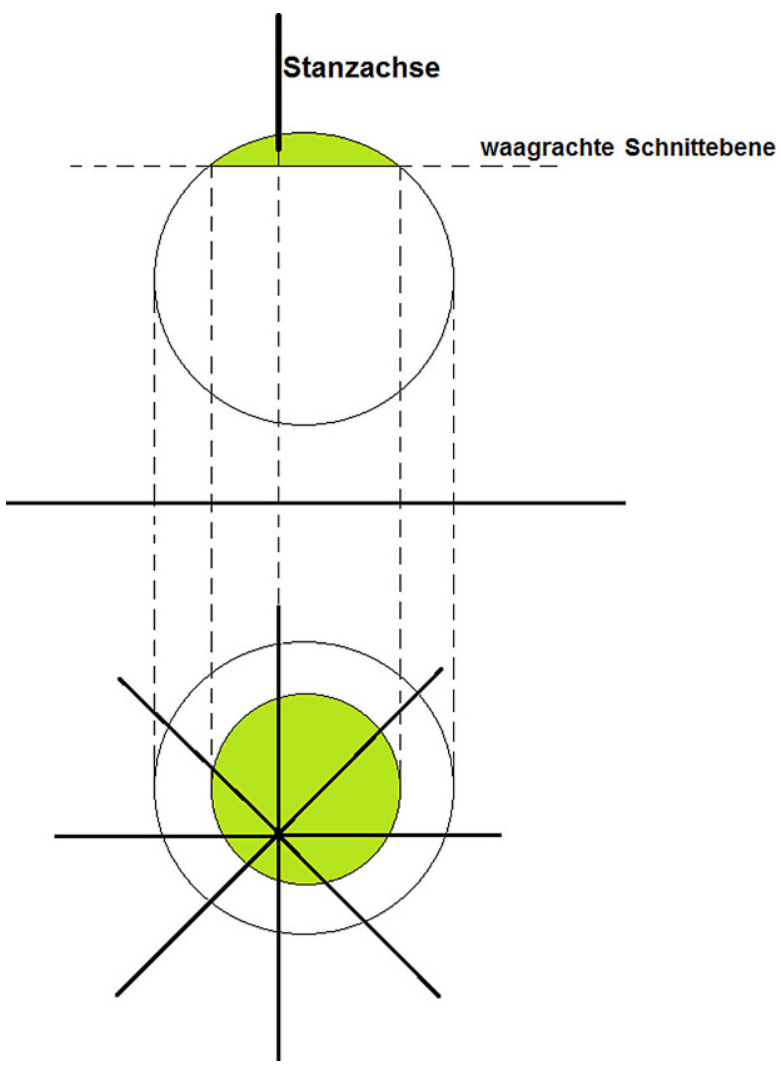

- Teile unterteilt), die man von oben lotrecht auf den Apfel drückt, wobei das Zentrum der Stanze irgendwo das „,im Norden“ abgetrennte Kugelsegment trifft. Anschließend färbt man die entstandene Fruchtoberfläche schachbrettartig (weiß und grau); dann gilt: Die Volumina aller grauen Teile zusammen sind genau so groß wie die Volumina aller weißen Teile zusammen. Analoges gilt auch für die Oberflächeninhalte. D. h. man hat eine perfekte Aufteilung unter 2 Personen.

In diesem Phänomen ist einerseits eine wirklich schöne Anwendung des Cavalieri-Prinzips zu sehen. Der Beweis des Pizza-Theorems ist nicht ganz einfach, aber diese Verallgemeinerung (Volumen) auf die Apfelteilung benötigt keine weiteren Rechnungen oder formalen Überlegungen mehr, es genügt das Prinzip von Cavalieri und die Idee einer weiteren waagrechten Teilung der Frucht, um die Pole ,entgegengesetzt gleich“ zu machen. Bei den Betrachtungen zur gerechten Teilung der Oberfläche kommt man andererseits auch ohne langwierige Rechnungen aus, wenn man sich die Oberfläche als ,unendlich dünne“ Hohlkugel vorstellt (die Gleichheit der Volumina überträgt sich dann auf die Gleichheit der Oberflächeninhalte; genauer siehe Anhang). D. h. bei beiden Phänomenen (Volumen, Oberfläche) kommt man ohne Rechnen und abstrakte bzw. streng formale Überlegungen zu schönen und vielleicht überraschenden Resultaten (wenn man das Pizza-Theorem kennt), und zwar durch Anwendung wichtiger Prinzipien bzw. Vorstellungen. 
Leider lässt sich eine gerechte Aufteilung unter mehr als 2 Personen so nicht erreichen, weil es nur zwei Polregionen gibt. In der Kugelschicht zwischen den beiden Breitenkreisen von $A$ und $B$ ließe sich das zwar leicht bewerkstelligen (mit einer Stanze, die jeden Quadranten in mehr als zwei gleichwinklige Teile teilt ${ }^{5}$ ), aber der einfache Trick mit dem Umfärben eines Poles funktioniert bei mehr als zwei Farben (Personen) leider nicht mehr.

Kugeln können mit GeoGebra (3D) auch leicht mit Ebenen geschnitten werden, so dass die entsprechenden Kreise (im Bild dann Ellipsen) und vor allem die entsprechenden Teile (krummlinige Dreiecke) der Kugeloberfläche sichtbar gemacht werden können.

Leider kann man die Volumina und Oberflächeninhalte der einzelnen Stücke nicht leicht ausrechnen, auch mit GeoGebra kann man deren Werte nicht einfach bestimmen, und die einzelnen Teile auch nicht unterschiedlich färben ${ }^{6}$, so dass man Bilder wie Abb. 3 nicht innerhalb von GeoGebra erzeugen kann. D. h. Experimente mit einer Dynamischen Geometrie Software ${ }^{7}$ als Messinstrument mit dem Ziel, dass Lernende dieses Theorem selbst entdecken, sind hier wohl wenig zielführend. Aber eine Aufgabe der folgenden Art könnte bewerkstelligt werden (z. B. in einer Lehrveranstaltung über Geometrie oder über Problemlösen): Wie kann man das PizzaTheorem nutzen, um das Apfel-Theorem zu zeigen? Dabei könnte - je nach Leistungsstand der Lernenden - auch ein Hinweis auf das Cavalieri-Prinzip erfolgen, die Ideen mit dem Umfärben einer Polregion muss dann immer noch selbständig beigesteuert werden. Für reale Experimente mit solchen Teilungen reichen Äpfel (Melonen, Kartoffeln) und ein großes scharfes Messer, um solche Teile auch physisch vor sich zu haben, und nicht nur als Gedankenobjekte.

Eine Recherche $\mathrm{zu}$ diesem Thema hat ergben, dass auf diese Idee auch schon andere Mathematiker kamen. So schreibt ${ }^{8}$ z. B. George Berzsenyi 1994 [1], dass dieses Phänomen von Michael Nathanson ${ }^{9}$ als Student entdeckt wurde, er nannte es offenbar „Calzone Theorem“ (dieser Name rührt vermutlich daher, dass in Amerika eine „Calzone“ oft wirklich kugelförmig ist, mit verschiedenen Käsesorten gefüllt. Wikipedia über „Calzone“: As a rule, calzones are usually stuffed with cheeses such as ricotta, mozzarella, Parmesan, provolone or a different local cheese. The dough is folded into a half-moon shape, then either sealed with an egg mixture or shaped into a sphere. It is then either baked or fried.):

Choose any point $P$ inside or on the boundary of a sphere (calzone), any line through this point, and four planes through this line making eight equal

\footnotetext{
5 Beim ebenen Pizza-Theorem kann man auch unter mehr als 2 Personen einfach gerecht teilen (vgl. [3, S. 182]).

6 Dieses unterschiedliche Färben der Teile ist mit tiefer liegenden Tricks auf relativ umständliche Art und Weise auch mit GeoGebra möglich ist. Für die Abb. 2 und 3 wurde zum Färben ein anderes Programm benutzt.

7 Neben GeoGebra gibt es auch noch andere wie Cindarella, SketchPad, etc.

8 Weitere Hinweise haben wir in der Literatur leider nicht gefunden.

9 Momentan Professor am St. Mary's College, Kalifornien. In einer privaten Email schrieb er, dass er nie einen Beweis zu diesem Theorem veröffentlicht, aber schon zahlreiche Vorträge zum Thema „PizzaTheorem“ und „Calzone-Theorem“ in den letzten Jahren gehalten hat.
} 
Abb. 6 Gerechte Aufteilung der Oberfläche (,Apfelschale“)

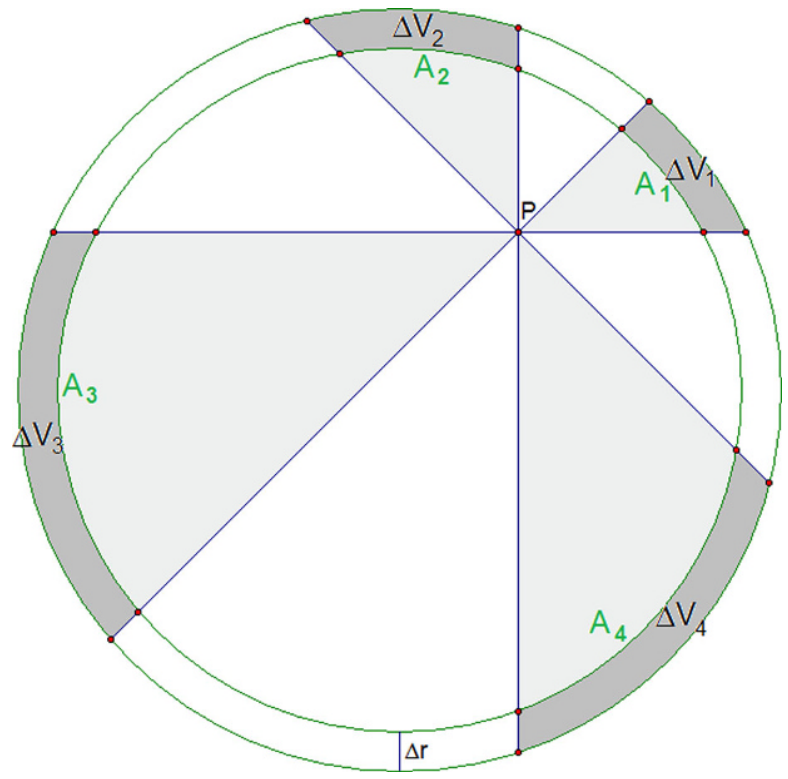

$45^{\circ}$ angles at $P$. Then these planes, together with the plane perpendicular through this line, divide the calzone into 16 pieces, which can be colored alternately black and white, so that the total volume of the black pieces will be equal to the total volume of the white pieces. The proof can be obtained by using Cavalieri's Principle.

Funding Open access funding provided by University of Vienna.

Open Access This article is distributed under the terms of the Creative Commons Attribution 4.0 International License (http://creativecommons.org/licenses/by/4.0/), which permits unrestricted use, distribution, and reproduction in any medium, provided you give appropriate credit to the original author(s) and the source, provide a link to the Creative Commons license, and indicate if changes were made.

\section{Anhang}

\section{Genauere Begründung der gerechten Teilung des Oberflächeninhalts (,Apfelschale ${ }^{6}$ )}

Durch das Umfärben einer Polregion ist garantiert, dass in den Polregionen insgesamt Flächengleichheit zwischen Grau und Weiß herrscht. Es bleibt noch genauer zu begründen, dass diese Flächengleichheit auch zwischen den beiden Breitenkreisen von $A$ und $B$ (siehe oben) gegeben ist. Wir werden sehen, dass die Argumentation analog zum ebenen Pizza-Theorem (vgl. [3, 186f.]) verläuft, nur ,eine Dimension höher“: Statt Bogenlängen und Flächeninhalten haben wir hier Oberflächeninhalte und Volumina. 


\section{Zwischen den beiden Breitenkreisen von $A$ und $B$}

Wir haben oben schon festgestellt (Prinzip von Cavalieri), dass - bei beliebigem Radius - das Volumen von Grau unabhängig von der Lage der Stanzachse (Punkt $P$ ) und von der (Rotations)-Lage der Messer ist, nämlich das halbe Gesamtvolumen. Daher gilt dies auch für die Differenz zweier solcher Volumina bei verschiedenen Radien, d. h. für die Volumensumme $\Delta V=\Delta V_{1}+\Delta V_{2}+\Delta V_{3}+\Delta V_{4}$ der vier grauen Hohlkugelteile bei Vergrößerung des Radius von $r$ auf $r+\Delta r$ (Abb. 6; dort allerdings leider nur in einem zweidimensionalen Querschnitt dargestellt; die Oberflächen $A_{1}, A_{2}, A_{3}, A_{4}$ erscheinen dort als Kreisbögen).

Da sich also offenbar $\Delta V \approx\left(A_{1}+A_{2}+A_{3}+A_{4}\right) \cdot \Delta r$ (die Hohlkugel hat ja überall dieselbe Dicke $\Delta r$, so dass man sich die Hohlkugelteile zusammen auch näherungsweise als Zylinder mit Grundflächeninhalt $A_{1}+A_{2}+A_{3}+A_{4}$ und Höhe $\Delta r$ vorstellen kann) bei Verschiebung bzw. Drehung des Stanzmessers nicht ändert (konstant ist), gilt dies auch für $\frac{\Delta V}{\Delta r}$ und im Limes auch für $\lim _{\Delta r \rightarrow 0} \frac{\Delta V}{\Delta r}=\frac{\mathrm{dV}}{\mathrm{d} r}=$ $A_{1}+A_{2}+A_{3}+A_{4}$ (Summe der vier grauen Oberflächeninhalte). D. h. auch die Summe der vier grauen Oberflächeninhalte $A_{1}+A_{2}+A_{3}+A_{4}$ (,Apfelschale“) ist unabhängig von der Lage des Punktes $P$ und der (Rotations-)Lage der Messer und damit beträgt sie die Hälfte der Gesamtoberfläche, die andere Hälfte ist weiß.

Im Kern geht es also darum, dass die Ableitung des Volumens einer Kugel - und zwar die Ableitung nach dem Radius - ihrem Oberflächeninhalt entspricht (analog entspricht die Ableitung des Flächeninhalts eines Kreises dem Umfang). Es ist sowohl geometrisch als auch didaktisch wichtig, nicht nur formal $\frac{\mathrm{d} V}{\mathrm{~d} r}=O$ bestätigen zu können durch Verwenden der entsprechenden Formeln $\stackrel{\mathrm{d} r}{V}=\frac{4 \pi}{3} \cdot r^{3}, O=4 \pi \cdot r^{2}$ bzw. beim Kreis $\left.A=\pi \cdot r^{2}, U=2 \pi \cdot r\right)$ und der bekannten Differentiationsregeln, sondern diese Zusammenhänge auch inhaltlich erklären zu können. Oben haben wir davon nicht für eine ganze Kugel Gebrauch gemacht, sondern nur für einen Teil (jene Kugelschicht zwischen den beiden Breitenkreisen durch $A$ und $B$, siehe oben).

\section{Literatur}

1. Berzsenyi, G.: The Pizza Theorem - Part II. Quantum 4(4), 29 (1994)

2. Heuser, H.: Lehrbuch der Analysis, Teil 2. BG-Teubner, Stuttgart (1983)

3. Humenberger, H.: Gerechte Pizzateilung - keine leichte Aufgabe! Math Semesterberichte 62(2), 173-194 (2015). https://doi.org/10.1007/s00591-015-0146-8) 